\title{
AN ENERGY BASED FOULING MODEL FOR GAS TURBINES: EBFOG
}

\author{
${ }^{1},{ }^{2}$ Nicola Casari, \\ Michele Pinelli, Alessio Suman \\ ${ }^{1}$ University of Ferrara \\ Via Saragat, 1 \\ Ferrara (IT), 44122
}

\author{
Luca di Mare, \\ Francesco Montomoli \\ ${ }^{2}$ Imperial College London \\ London (UK), SW7 2AZ
}

\section{ABSTRACT}

Fouling is a major problem in gas turbines for aeropropulsion because the formation of aggregates on the wet surfaces of the machine affects aerodynamic and heat loads.

The representation of fouling in CFD is based on the evaluation of the sticking probability, i.e. the probability a particle touching a solid surface has to stick to that surface. Two main models are currently available in literature for the evaluation of the sticking coefficient: one is based on a critical threshold for the viscosity, the other is based on the normal velocity to the surface. However, both models are application specific and lack generality.

This work presents an innovative model for the estimation of the sticking probability. This quantitiy is evaluated by comparing the kinetic energy of the particle with an activation energy which describes the state of the particle. The sticking criterion takes the form of an Arrhenius-type equation. A general formulation for the sticking coefficient is obtained. The method, named EBFOG (Energy Based FOulinG), is the first "energy" based model presented in the open literature able to account any common deposition effect in gas turbines.

The EBFOG model is implemented into a Lagrangian tracking procedure, coupled to a fully three-dimensional CFD solver. Particles are tracked inside the domain and equations for the momentum and temperature of each particle are solved. The local geometry of the blade is modified accordingly to the deposition

\footnotetext{
*Address all correspondence to this author, email: nicola.casari@unife.it, nicola.casari15@imperial.ac.uk
}

rate. The mesh is modified and the CFD solver updates the flow field.

The application of this model to particle deposition in high pressure turbine vanes is investigated, showing the flexibility of the proposed methodology. The model is particularly important in aircraft engines where the effect of fouling for the turbine, in particular the reduction of the HP nozzle throat area, influences heavily the performance by reducing the core capacity. The energy based approach is used to quantify the throat area reduction rate and estimate the variation in the compressor operating condition. The compressor operating point as a function of the time spent operating in a harsh environment can be in this way predicted to estimate, for example, the time that an engine can fly in a cloud of volcanic ashes. The impact of fouling on the throat area of the nozzle is quantified for different conditions.

\section{NOMENCLATURE}

A Pre-exponential constant

$c$ Specific heat

$C_{D} \quad$ Drag coefficient

$C_{1}$ Activation energy - Constant part

$C_{2}$ Universal constant of the reduced temperature

$d_{p}$ Particle diameter

$e$ Gas specific energy

$E_{\text {act }}$ Activation energy

$E_{\text {case }}$ Reference energy for the case

$F_{B}$ Brownian force 
$F_{D} \quad$ Drag force

$F_{S} \quad$ Saffmann force

$\dot{m}$ Mass flow rate

$m_{p} \quad$ Particle mass

$p$ Pressure

$R e_{p} \quad$ Particle Reynolds number

$S_{p} \quad$ Sticking probability

U Fluid velocity

$T$ Temperature

$T * \quad$ Reference Temperature

$u_{p}$ Particle velocity component

$u$ Fluid velocity component

$x$ Physical coordinate

$y$ Physical coordinate

\section{Greek letter symbols}

$\alpha$ sum of squared first order derivatives respect to $\eta$

$\beta$ sum of product of first order derivatives

$\gamma$ sum of squared first order derivatives respect to $\xi$

$\eta$ computational coordinate

$\xi$ computational coordinate

$\mu \quad$ Fluid viscosity

$\rho$ Gas density

$\rho_{p} \quad$ Particle density

\section{INTRODUCTION}

Solid particles can enter the power stream of gas turbines for different reasons. Particles can be ingested during take-off or landing in harsh environments, such as sand storms [1], or during cruse at altitude through volcanic dust clouds [2]. Solid particles can also be produced during the combustion of heavy oils or synfuels and even the composition of the ingested particle can change during the passage through the combustion chamber [3]. Coal gasification also produces small amounts of particulate, which can be reduced, but never eliminated [4].

The ingestion of solid particles can deteriorate the performance and stability of gas turbines through the erosion of blades and annuli and through the build-up of deposits on the aerofoils. In some cases, the effect of the ingested particles is sufficient to demand engine shut-down or to cause outright failure $[1,2]$. Dunn [5] estimated that for power plants with TET (Turbine Entry Temperature) below $1283 \mathrm{~K}$, the main cause of damage is erosion in the compressor, while for power plants with TET above this value, failure is caused by foulant deposition in the hot components.

Particles sticking on the first stage nozzle of the high pressure turbine result in an increase in aerofoil thickness and roughness. The uncontrolled modification to the blade shape and roughness invariably brings about an increase in loss. Foulant deposits can also clog cooling holes. In the most severe cases, this leads to a reduction in life due thermal stresses, local overheating and creep [6]. Finally, the increased boundary layer displacement thickness - due to the increased roughness and uncontrolled change in shape - and the build-up of foulant [7] can cause a reduction in passage area and hence a drop in turbine capacity. This, in turn, can push the compression system beyond its stability limit, making the engine inoperable. Even if no immediate mechanical damage is suffered, once fouling takes place, the removal of foulant deposits is very time consuming and expensive: minimizing the deposition can reduce the time spent cleaning the engine and prolong the hardware's life.

There is a strong need, therefore, to perform research aimed at avoiding or reducing fouling because of its negative impact on performance, reliability and operability. This need is made even stronger by the possible introduction of fuels derived from alternative sources [8].

Understanding the mechanism by which particles stick to the walls and the conditions which favour or counter-act fouling is of fundamental importance to the prediction of deposition rates. All the models developed for the computation of fouling phenomena in CFD are based on the definition of the sticking coefficient: this is an index of the likelihood that a particle impinging onto a surface sticks to that surface.

In models based on critical viscosity [9], the sticking coefficient is linked to the viscosity of the particle and it is therefore dependent on the temperature and chemical composition of the foulant agent. It is assumed that the sticking coefficient approaches unity at the softening temperature of the fouling agent. Further development of this model [10] takes into account the momentum loss, quantified by the coefficient of rebound. The main limitation of this model is that the dependence on impact angle, impact velocity and particle mass is neglected. Recently published work by Singh et al. [10] overcomes some of these limitations. However, the empirical assumptions make the model of difficult generalization. In addition, the model for the viscosity prediction by [11] is suitable only for a small range of materials.

The critical velocity model by [12] is based on the impact velocity component normal to the surface. This model requires that the elastic properties of both foulant agents and surfaces materials to be known. A feature of the model is that it predicts a reduction in capture velocity for increasing Young's moduli of the foulant or surface: sticking probabilities decrease with higher stiffness. Unfortunately this model does not account for the stickiness of molten or soften particles (capillary forces), which are often higher than other attractive forces.

There are three possible models/implementations for the computation of the particle laden flows. These three models are representative of different concentrations of the discrete phase [13]. At low concentrations, the particles have negligible effect on the flow field. This situation is represented via this one-way coupling. This means that the only momentum is transferred from the fluid to the particles and not vice-versa: the momen- 
tum transfer from the particles to the fluid has an insignificant effect on the flow. A second model, for higher concentration, considers that the momentum transfer from the particles is large enough to modify the flow and turbulence structures. This second way of interaction is called two-ways coupling. Flows in these two regimes (one-way and two ways) are often referred to as dilute suspensions. The third regime with very high concentration, is often called dense suspensions. In addition to the two-way coupling between the particles and the flow, the particle/particle collision effect must be taken in account. This way of interaction is often termed four-way coupling. A more extensive description of all the coupling can be found in [13]. The authors carried out an extensive explanation of all different regimes with the concentration threshold for the passage from a regime to the next one.

Each of the coupling methods can be implemented within an Eulerian or a Lagrangian approach. The Eulerian formulation considers both gas and particulate flows as continuum media and the phases are treated as two interacting fluids. The main advantage of the Eulerian formulation is the faster computation. In addition, effects of interactions, particularly turbulence, between two phases (two-way coupling) are easily evaluated.

The main disadvantage of the Eulerian formulation is that it gives mean values of the particulate phase over a small control volume: there is little information about particles trajectories and their actual velocity. For this reason, it is confined to the higher concentration, where the Lagrangian approach would be computationally prohibitively expensive.

In the Lagrangian approach, the motion of each particle is considered and particle trajectories are evaluated explicitly. A momentum balance is solved for each particle. However, as already pointed out, this method is computationally expensive: the trajectories of a great number of particles must be tracked. The flow field of the carrier flow is obtained through an Euler or Navier Stokes calculation, and then the trajectories of individual particles are computed by integrating the force balance equation for each particle (drag and lift forces due to the particle size, shear stress, rotation, diffusion and flow temperature).

The first experimental studies to evaluate the extent of turbine deposition was carried out by [14]. Later studies have assessed the influence of particle velocity, temperature and size on deposition rate. Delimont [15] considered sand deposition on a coupon at a prescribed approaching. Crosby [16] studied fly ash deposition on specimens at varying gas temperature. Dykhiozen [17] and Legoux [18] studied deposition efficiency in cold spray varying the velocity and the temperature, respectively.

The aim of this work is to provide a general model which can easily be implemented in every CFD code, to take into account the following contributions to fouling: particle, gas and wall temperature, particle size, particle velocity and particle composition. In order to do this the new model has been conceived as an energy based approach: as the energy involved in the impact in-

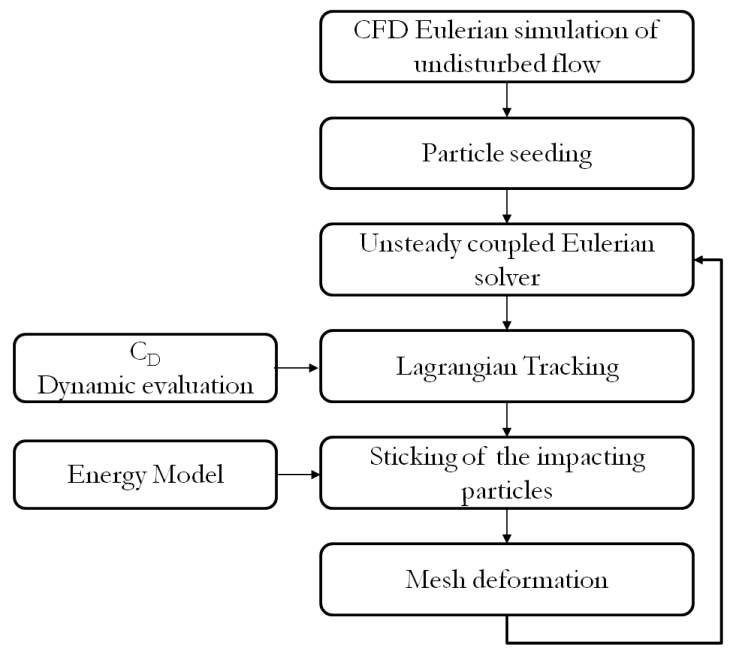

FIGURE 1. Computational procedure

crease, the sticking probability rises. The new approach shows good agreement with all the above mentioned cases. This model is based on an appropriate form of the Arrhenius equation which compares the energy of the particle/surface interaction (given by the sum of kinetic energy flux normal to the surface) with a threshold energy value (activation energy). If the impact involves an amount of energy greater than the activation energy the sticking process takes place. Erosion is not considered in this work, even though the model can be easily adapted to keep in account this phenomenon.

Furthermore several application of this model to different kind of particle are presented. The final results of this paper are presented as a stability map for the engine (before the flame out) when the aircraft flies through a cloud of particles (such as volcanic ashes). The maximum flight time allowed depends on the density of the cloud and the accretion rate of the deposit.

\section{METHODOLOGY}

The procedure to compute fouling effect on turbomachinery performance follows the outline in Figure 1. The model starts with a steady CFD solver that evaluates the flow field in the absence a solid phase. Once convergence is achieved, the flow is seeded with particles and conservation equations for the two phases are marched to convergence concurrently. Two kinds of simulation have been carried out: one with one-way interaction (particle trajectory driven by the flow field) and one with two-way interaction (particle affecting the flow field [13]). The differences in the two approaches are explained in detail below.

From the flow velocity and the drag coefficient $C_{D}$ of the particles (evaluated according to [19]) a Lagrangian tracking code evaluates the particles trajectory with a second order integration of Newton's momentum equation. The particle tracking method 


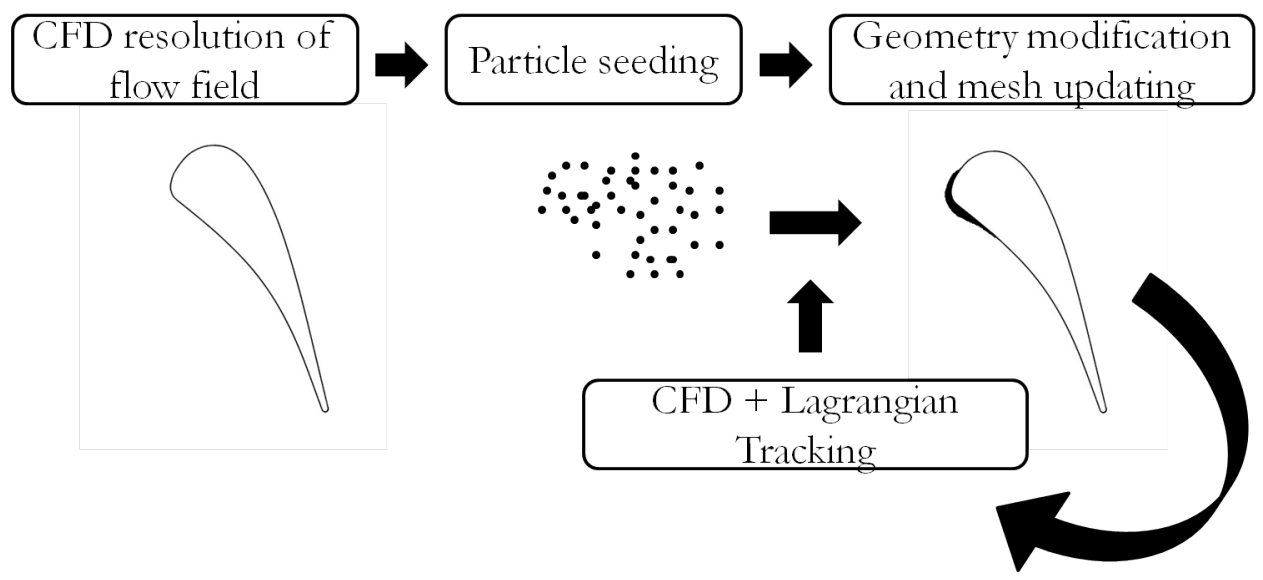

FIGURE 2. Sticking evaluation procedure

is based on the jump-and-walk algorithm proposed by [20]. Particle tracking is performed on a tetrahedral grid obtained by splitting the hexahedra of the CFD grid. The tracking implementation is fully parallel, as the CFD solver [21], and relies on message passing libraries (MPI).

When a particle hits the blade surface the energy based model evaluates the sticking probability. The proposed model is based on kinetic energy associated to the velocity component normal to the wall, the temperature, particle size, and composition. If the particle sticks to the surface, a mass balance is performed on the foulant deposit to determine its growth in thickness and the geometry is modified accordingly. The mesh and the flow field are finally updated. The particles affect the flow field altering the shape and roughness of the wall, the boundary layer structure, the shock intensity. It must be remarked that the effect of the roughness is not present in the simulations shown in this work because the mesh elements are bigger than the average length scale that characterizes the roughness. Therefore roughness changes during the deposition process and their effect on the fluid flow have not been taken into account. A denser mesh would autmoatically resolve such effects.

The particle deposition in the first stage of a nozzle of an high pressure turbine, LS89 profile (see [22]), has been investigated and simulated. In the following paragraphs the new approach towards deposition and fouling is explained, both in its physical outline and in its implementation: see Figure 2.

\subsection{CFD Euler simulation of the undisturbed flow.}

The simulation of the flow in the passage in absence of fouling agent has been carried out with an in house CFD solver [21] validated for turbomachinery applications. The solver is exercised on a C-type grid. The mesh is first generated by transfinite interpolation. Then elliptic smoothing 1 based on a Laplace equation is performed [23]:

$$
\begin{aligned}
& \alpha x_{\xi \xi}-2 \beta x_{\xi \eta}+\gamma x_{\eta \eta}=0 \\
& \alpha y_{\xi \xi}-2 \beta y_{\xi \eta}+\gamma y_{\eta \eta}=0 \\
& \alpha=x_{\eta}^{2}+y_{\eta}^{2} \\
& \beta=x_{\eta} x_{\xi}+y_{\eta} y_{\xi} \\
& \gamma=x_{\xi}^{2}+y_{\xi}^{2}
\end{aligned}
$$

The relationships (1) describe a smooth distribution of computational coordinates $(\xi, \eta)$ in physical space $(x, y)$. The set of equations (1), derives from a Laplacian operator applied to the computational coordinates,as shown in Eqn. (2). Since the unknowns are the coordinates in the $(x, y)$ space, the set of equations that project the computational space into the physical one is the finite difference implementation of Eqn. (1).

$$
\nabla^{2} \eta=0 \quad \nabla^{2} \xi=0
$$

\subsection{Particle seeding}

Once the undisturbed simulation reach the convergence, the flow is seeded with particles with given size distribution. The particles seeding inside the computational domain is done by spreading a fixed amount of particles in each cell. The particles distribution, size and concentration are chosen according to Taltavull [24]. Taltavull [24] has shown that the size distribution of the particulate reaching the turbine is very different from the one ingested at the intake. The causes for this change are basically two: the large particles are centrifuged towards the bypass air flow and part of those sent into the core are fragmented by the impact with compressor blades. The result of these interactions is that it is unlikely that particles larger than $30 \mu m$ can reach the turbine vanes. 
The foulant agent used in this work is representative of Southern Iceland volcanic ash data, and in particular of the Laki volcano (exploded in 1784) deposits. The Laki ash is richer in iron and is likely to have a higher melting temperature than the ash erupted by the Eyjafjallajökull volcano in 2010 [25], causing widespread disruption to european air traffic. Taltavull [24], however, showed that the deposition rates for the Laki ash are similar to those recorded for the Eyjafjallajökull ash.

The specific heat of the material found by [24] is approximately $800 \mathrm{~J} \mathrm{~kg}^{-1} \mathrm{~K}^{-1}$, while its density is roughly $3 \mathrm{~kg} \mathrm{~m}^{-3}$. By following the distribution suggested by [24] the particle sizes are distributed uniformly between $0.1 \mu \mathrm{m}$ and $30 \mu \mathrm{m}$.

\subsection{Quasi-Unsteady One/Two Way Coupling with Eu- lerian Solver}

Depending on the particle concentration, the effect that the particles have on the fluid needs to be evaluated. In this article both the coupled and uncoupled method are used. The difference between the two approaches are reported below.

From the physical standpoint, the differences between the two methods lies in the correction of the conservation equations. In particular (since the variation of mass is not allowed in this article), the conservation equations for the flow field (3)

$$
\frac{\partial}{\partial t}\left(\begin{array}{c}
\rho \\
\rho \mathbf{U} \\
\rho e
\end{array}\right)+\nabla \cdot\left(\begin{array}{c}
\rho \mathbf{U} \\
\rho \mathbf{U} \otimes \mathbf{U}+p \mathbf{I} \\
\rho \mathbf{U} h
\end{array}\right)=\left(\begin{array}{l}
0 \\
0 \\
0
\end{array}\right)
$$

must be modified and becomes:

$$
\frac{\partial}{\partial t}\left(\begin{array}{c}
\rho \\
\rho \mathbf{U} \\
\rho e
\end{array}\right)+\nabla \cdot\left(\begin{array}{c}
\rho \mathbf{U} \\
\rho \mathbf{U} \otimes \mathbf{U}+p \mathbf{I} \\
\rho \mathbf{U} h
\end{array}\right)=\left(\begin{array}{c}
0 \\
\Sigma_{i} \mathbf{F}_{D i} \\
\Sigma_{i} H_{i}
\end{array}\right)
$$

In the one way coupling, the particle has no effect on the fluid [13] both in terms of momentum and energy transfer between the phases. Thus the problem of the computation of the flow and thermal fields can be solved in its classical form (3).

In the two way coupling the transfer of energy and momentum from the solid phase to the fluid phase is computed. In this case the system of equations to be considered is the (4). The meaning of the terms on the right hand side of the Equations (4) is the following:

- The first term is the mass variation due to the particle-flow interaction. Since evaporation or condensation are not allowed in this treatise, this term is equal to zero.

- The second one is linked to the momentum transferred from the flow to the particle or vice versa. It can be either positive (and tough the particle receive momentum from the fluid) or negative (the particle lose momentum in favor of the fluid). This happens for example in decelerating fluids or through shock waves. In general, this term is indicated with $\Sigma_{i} \mathbf{F}_{D i}$, where $\mathbf{F}_{D i}$ represents the drag force acting on the $\mathrm{i}$-th particle contained in the cell.

- The last term represent the transfer of energy between the two phases. This energy can be transferred in form of work done by the forces on the particles, and though $\sum_{i} \mathbf{F}_{i} \cdot \mathbf{u}_{p i}$, where $\mathbf{u}_{p i}$ is the velocity of the $\mathrm{i}$-th particle contained in the cell. Furthermore, a second kind of energy transfer may be present: the contact between particle and fluid allows the two phases to exchange heat. An additional term can be considered though: $\Sigma_{i} c_{i} \frac{d T_{i}}{d t}$. The last term of equations is $\Sigma_{i} H_{i}$, with the meaning of Eqn. (5)

$$
\Sigma_{i} H=\Sigma_{i} \mathbf{F}_{i} \cdot \mathbf{u}_{p i}+\Sigma_{i} c_{i} \frac{d T_{i}}{d t}
$$

A more extensive explanation of the forces acting on the particle is shown in the next paragraph.

1.3.1 Model for the discrete phase The trajectories of the discrete phase are computed with a Lagrangian balance on each particle. The equation describing such motion is Eqn. (6).

$$
\frac{d \mathbf{u}_{p}}{d t}=\mathbf{F}_{D}+\frac{g\left(\rho_{p}-\rho\right)}{\rho_{p}}+\mathbf{F}_{S}+\mathbf{F}_{B}
$$

An extensive description of each term of the force balance (Drag forces, Saffman forces, Brownian forces and buoyancy) can be found in [26]. In this article the Eqn. (6) is reduced to:

$$
\frac{d \mathbf{u}_{p}}{d t}=\mathbf{F}_{D}
$$

The particle motion is affected by the motion of the carrier phase through the drag force only. The other forces are neglected because are at least one order of magnitude lower than drag in the range of particle size considered (see [27]).

The drag force $F_{D}$ is evaluated as

$$
\mathbf{F}_{D}=\frac{18 \mu}{\rho_{p} d_{p}^{2}} \frac{C_{D} R e_{p}}{24}\left(\mathbf{u}-\mathbf{u}_{p}\right)
$$

where $\mu$ is the fluid viscosity, $d_{p}$ is the particle diameter and $R e_{p}$ is the particle Reynolds number defined as 


$$
R e_{p}=\frac{\rho d_{p}\left|u_{p}-u\right|}{\mu}
$$

The drag coefficient $C_{D}$ is evaluated iteratively using to the correlations given by [19]. The relation given by [19] for the evaluation of the drag coefficient is valid for perfectly spherical particles. As stated by [24], the shape of the typical volcanic ash is far from being spherical. In this work, the assumption of considering an equivalent diameter for non-spherical particle has been introduced. A time buffer between the seeding of the particles and the sticking evaluation is required in order to let the particle reach their actual velocity. A test to evaluate the impact of particles in the free stream on shock structures has been carried out. With the concentration and particle sizes specified in this work a weak impact on shock structures is observed (without considering the deposition and subsequent geometrical variation).

\subsection{Lagrangian Tracking}

In the evaluation of the exchange terms between the solid phase and the carrier phase, the properties of the fluid phase are those pertaining the cell occupied by each particle. As each particles moves through the computational domain it is necessary to identify the index of the cell where it resides. This is achieved through the Lagrangean tracking algorithm. For the purpose of tracking, a second representation of the computational domain is generated. In this representation each hexahedron forming the computational grid is split into tetrahedra. The reason of doing so is that it is easier to test whether a particle is inside inside or outside a tetrahedron than wheter it is inside or outisde a hexahedron.

The particle tracking is performed with the jump and walk algorithm by [20] and described here briefly. The search starts from the element where the particle is located before evaluating the movement (it can be seen as the initial condition from the tracking standpoint). Once the calculation of the new particle position is performed, the isoparametric coordinates of the new position with respect to the initial cell are evaluated. If all coordinates are in the interval $[0,1]$, the search stops and the particle is attributed to the current cell. If the particle falls outside the element, the search continues in the neighbouring element corresponding to the smallest isoparametric coordinate. This algorithm is very computationally efficient and, for the numerical parameters employed in the present study, each particle is relocated on average in no more than three steps. The solution of the discrete phase and the tracking of the particles are sequential: firstly the Lagrangian force balance is solved and then the tracking of the particle is performed, once the final position is known.
This allows the code to evaluate whether the particle crosses the boundary of the domain.

\subsection{Sticking of impacting particles, EBFOG model}

When a particle hit the blade surface the energy based model evaluates the sticking probability. The proposed model investigates the deposition process under a statistical mechanics perspective: during the impact the velocity and the temperature of the particle are involved. These features are the two sides of the same coin, being both a measure of the energy content, respectively thermal and kinetic. Starting from this idea it has been decided to consider the whole phenomenon from an energetic standpoint. The energy-based model is implemented through an Arrhenius-type equation. This equation in its general form, $k=A e^{-\frac{E_{a c t}}{R_{g} T}}$, links the reaction rate to the temperature. In chemical reactions it considers that the higher the kinetic energy of two impacting particles, the higher the rate of the reaction.

This type of equations is used for the evaluation of the sticking process in heat exchangers. The initial rate of deposition (usually named crystallisation fouling) is driven by the concentration of the foulant agent. To foresee the accretion rate an Arrhenius type equation is used [28]. In this article a similar equation is used to evaluate the deposition on the blades of an high pressure turbine. Basically, the denominator of the exponential is the specific thermal energy. So the equation can be read as a comparison between energies. Thus adding the velocity and the mass to the temperature effect, a simple relation can be worked out and used to predict the sticking probability. Particularly, the effect of the dimension and the velocity is included in the kinetic energy. Hence its flux in the normal to the surface direction is added to the thermal energy at the exponential denominator. The exponential is the comparison between the activation energy and a reference energy for the case though.

$$
S_{p}=A e^{-\frac{E_{a c t}}{E_{\text {case }}}}
$$

Where $S_{p}$ is the sticking probability, $E_{a c t}$ is the activation energy of the process and $E_{\text {case }}$ is the reference energy for the case. This energy is comprehensive of both the kinetic and thermal terms. In particular, the temperature influences the activation energy of the process: the higher the temperature the lower the activation energy. This remark can be formalised expressing the exponential ratio in the following way:

$$
\frac{E_{a c t}}{E_{\text {case }}}=\frac{C_{1}}{\frac{1}{2} m_{p} v_{p, n}^{2}\left(1+C_{2} \frac{T}{T^{*}}\right)}
$$

Where $T^{*}$ is a certain temperature which cause the physical properties of the material to change. It is the softening temperature 
if the particle material is polycrystalline (e.g. ash, coal, sand) or the melting point if the material is a pure substance. In Eqn. (11) the influence the temperature has on the activation energy is kept into account. In particular it is thought to affect the value of the constant $C_{1}$ through the Taylor expansion (12) truncated at the first order:

$$
E_{a c t}(T)=\frac{C_{1}}{\left(1+C_{2} \frac{T}{T^{*}}+\cdots\right)}
$$

The constant $C_{2}$ is a universal non-dimensional constant (it is the same for every materials) and it is equal to 3027: this value has been found through a multiparametric fitting procedure. In this way, thanks to a parametric fitting, the value of $E$ and $A$ can be extracted from the experimental data.Their value are thought to be dependent on the chemical composition of both wall and particle. Further studies are required to better understand the relation between these parameters and the conditions of the particle. In this sense this model can be considered a generalisation of the JKR model [29]. The difficulties the usage of the analytical JKR model implies ( prediction of the variation of the elastic properties of the material with the temperature, evaluation of the surface energy and effect of the impact) are overcome by EBFOG. By using the experimental data to obtain the model constants, it can be shown that the model is generally applicable. Eventually, to obtain the probability an incoming particle has to stick to the blade surface, the Eqn. 13 should be used.

$$
S_{p}=A e^{-\frac{C_{1}}{\frac{1}{2} m_{p} v_{p, n}^{2}\left(1+C_{2} \frac{T}{T^{*}}\right)}}
$$

This is the fundamental assumption of the Energy Based Fouling model (EBFOG).

It must be remarked that the model to take into account the variation of composition of the blade surface during the deposition. In fact at the beginning of the simulation the blade is clean, but once a layer of deposit is formed, the material which covers the blade in the deposition area is the same of the incoming particles. For this reason the chemical interactions between the materials will be different in the two cases. The change in the activation energy of the process has not been taken into account, because few experimental data are available for the variation of the deposition rate with the exposure time but the proposed model can account this phenomenon.

\subsubsection{Evidence of model validity based on litera-} ture Several cases available in the literature have been investigated to prove the validity of the model. In the logarithmic graph

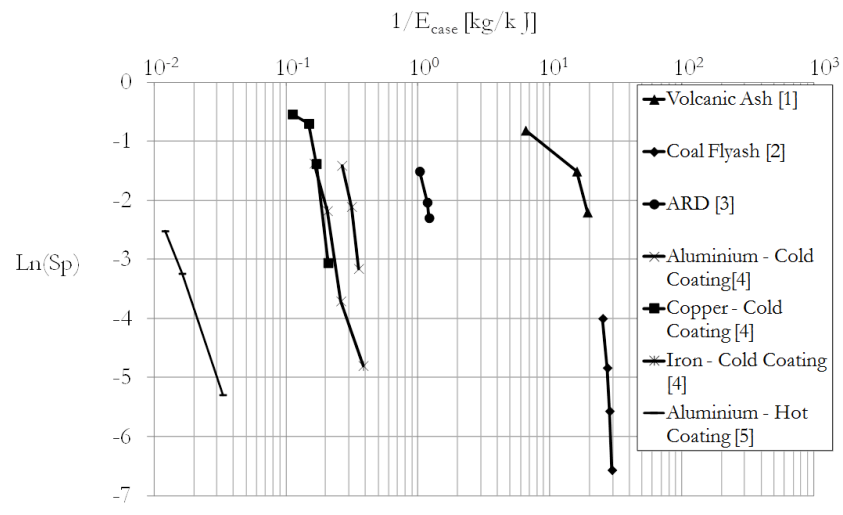

FIGURE 3. Comparison between different cases. References:1 [24], 2 [16], 3 [15], 4 [17], 5 [18]

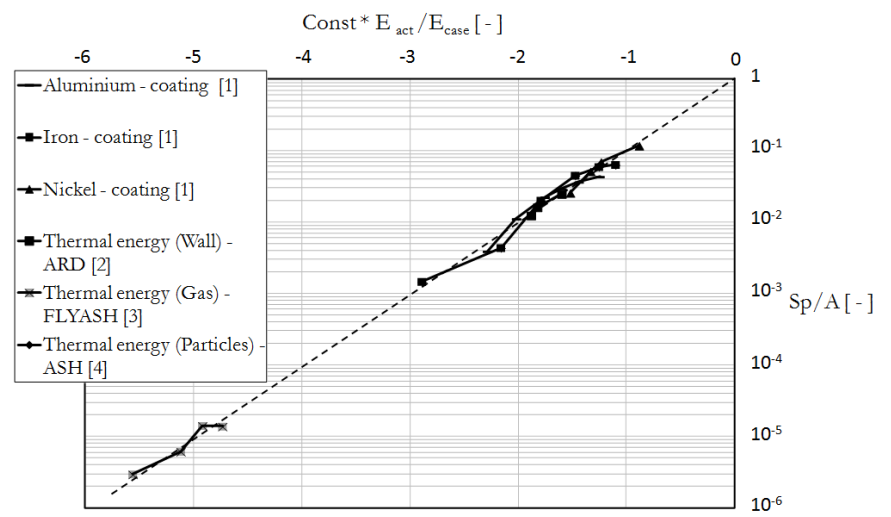

FIGURE 4. Comparison between different cases. References:1 [17], 2 [15], 3 [16], 4 [24]

of Figure 3 the linear trend of sticking probability with the reciprocal of the characteristic energy of the case is shown. The experimental data are taken from sand deposition on coupons at a prescribed approach velocity [15], fly ash deposition on specimens varying gas temperature [16], deposition in cold spray coating processes [17] and coating in higher temperature processes [18].

Although the curves are representative of very different cases, the trend is similar. Adjusting the parameters in such a way that the curves becomes dimensionless and plotting the different cases in a logarithmic graph, all the cases of figure 4 collapse on a line.

If the groups of quantities which are related to the temperature (reduced temperature) are separated from the rest, in the Eqn. 13, the graph represented in Figure 5 is obtained. A proof of the existence of a universal law for the energy variation with the reduced temperature is therefore achieved. By using Eqn. (12) the same trend is shown. The depicted curve (where all the curves collapses) is the universal trend of the activation energy 


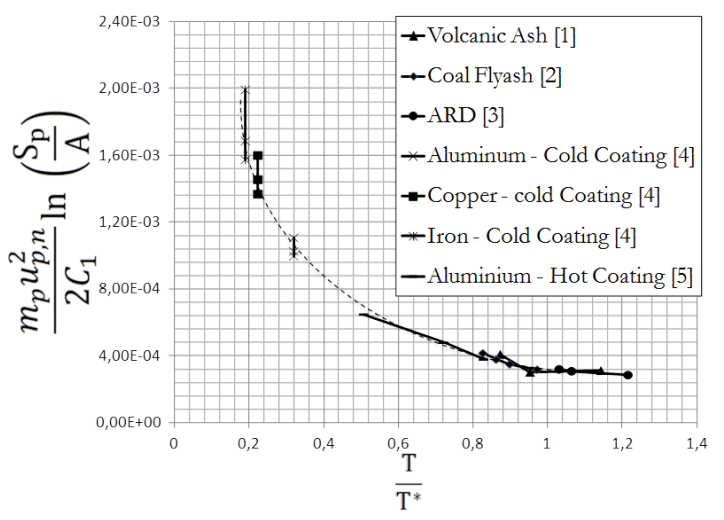

FIGURE 5. Universal law for the Activation energy variation with the temperature. References: 1 [24], 2 [16], 3 [15], 4 [17], 5 [18]

variation with the temperature. It is a rational function of the reduced temperature.

1.5.2 Boundary accretion Particles deposition is a stochastic process which depends on the sticking probability. Particle energy is evaluated and, through the exponential comparison (10), the sticking probability corresponding to that energy value is estimated. To keep into account the contribution of each particle and to avoid problems such as the lowering of particle concentration, a Metropolis-Hasting algorithm is introduced. This algorithm belongs to the family of the Markov chain Monte Carlo methods (MCMC), and it is used to reject some of the proposed moves in a random walk process. In this case it provides a useful tools for the selection of the hitting particles, making the probability value to be globally respected.

The deposition of the particles on the surface determines the formation of deposits. It must be remarked that once a first layer of deposit is formed, the material of the surface changes. It is reasonable to expect a change in the $E_{a c t}$ and in the roughness of the wall in the deposition area. These aspects have not been considered in this work, mainly for lack of experimental data and mesh size. However a denser mesh can show a local increment in roughness, without altering the algorithm (even if in this case a full Navier Stokes solver needs to be used for the flow field evaluation). The accretion of the deposit has been built as normal displacement of the surface at the impact point.

\section{Results}

In this section the effects of the exposing a VKI - LS89 blade to the particle laden fluid are shown. Particularly two cases are analysed: the case of volcanic ash, as analysed by [24], with composition specified in the previous paragraphs, and the case

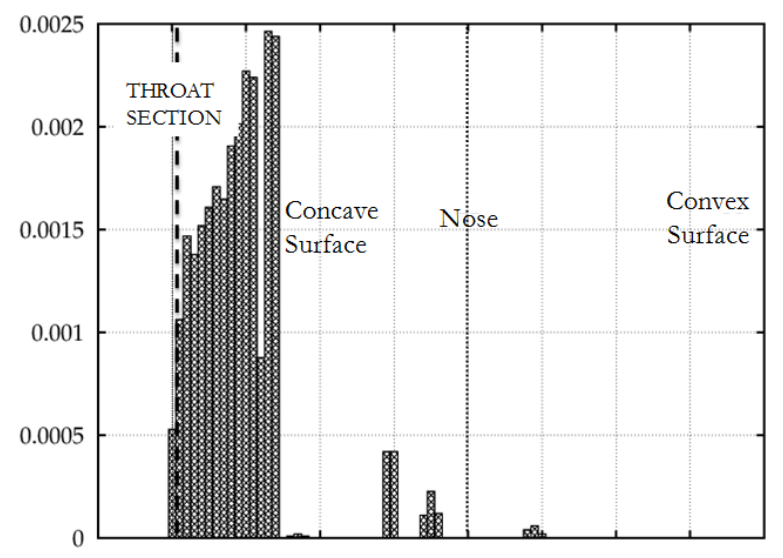

FIGURE 6. Thickness distribution on the LS89 VKI blade - particle size $1 \mu m$ at $T=1800 \mathrm{~K}$

of sand [15] cloud. These two cases consider the impact of a volcanic or sand cloud during flight conditions. The difference on the shape of the deposition between these two cases and the effect on the performance are therefore pointed out.

The results below are obtained using the boundary conditions provided by [22], scaling the data to the corresponding typical values of a modern turbine. In this work the Turbine Inlet Temperature (TET)is assumed to be $1800 \mathrm{~K}$ and the static pressure at the inlet of the domain is assumed to be $10^{6} \mathrm{~Pa}$.

\subsection{Geometry modification}

Quantitatively speaking the particles whose size is small (i.e. little or equal to $5 \mu \mathrm{m}$ ) tend to stick to the zone of the pressure side close to the trailing edge. This is because these particle have a small inertial effect and are able to follow quite entirely the fluid flow. The deflection from the streamlines happens only in the last part of the vane. The distribution of the deposit thickness along the blade (in normal to the surface direction) is shown in Figure 6. If the particle size is bigger (i.e. above $20 \mu \mathrm{m}$ ) the location of the impacts and of the deposition is shifted towards the leading edge. The variation of deposit thickness along the blade is reported in Figure 7.

From these graphs the variation of the throat area can be predicted. In this blade the throat area is very close to the trailing edge, being the duct simply convergent. The nominal minimum distance between two blades is $13.7 \mathrm{~mm}$. The rate of variation of the throat area is evaluated for different concentration of the dispersed phase, for different particles material and for several ratio $T E T / T_{\text {soft }}$. The results of these simulations are reported in Figure 8.

The $y$-axis in the previous graphs represent the rate of accretion of the build-up in the normal to the surface direction which 


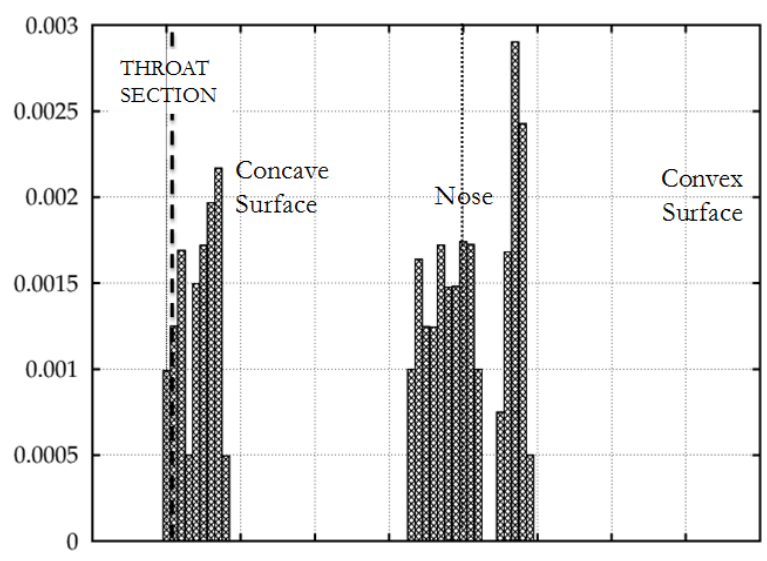

FIGURE 7. Thickness distribution on the LS89 VKI blade - particle size $25 \mu \mathrm{m}$ at $T=1800 \mathrm{~K}$

corresponds to the throat section trace on the plane in this case. The unity of measurement is $\mathrm{mm} / \mathrm{s}$ and though from this graph the reduction in the throat area can be derived.

\subsection{Area Variation due to Fouling}

The impact of area reduction on the jet engine performance is based on the fact that the flow processed by the compressor must be ingested by the turbine. So, in terms of non-dimensional mass-flow,

$$
\frac{\dot{m}_{1} \sqrt{T_{01}}}{p_{01}}=\frac{\dot{m_{3}} \sqrt{T_{03}}}{p_{03}} \frac{p_{03}}{p_{01}} \sqrt{\frac{T_{01}}{T_{03}}} \frac{\dot{m}_{1}}{\dot{m_{3}}}
$$

Where the subscript 1 stands for the inlet of high pressure compressor, 3 for the high pressure turbine section. The subscript 0 stands for the total value of the physical quantity. By assuming that the bleeds mass flow in the compressor are equal to the fuel mass flow $\left(\dot{m}_{1}=\dot{m}_{3}\right)$, the last term on the right hand side of the Eqn. (14) can be ignored.

A second assumption can be made: assuming that the cloud of foulant is encountered while the aircraft is flying at cruise velocity, the working point of the compressor is known and it is considered to be the design point. A common design assumption is that the design point lies on the line locus of the maximum efficiency points. This line is really close to the surge line, thus the risk of displacement of the working point beyond the surge line is not negligible in case of mass flow variation. In order to avoid this occurrence, a control system which prevent the point to cross the stall margin line (locus of the points whose distance from the surge line is equal to $2 \%$ of the adimensional flow) is always present. In case the working point crosses the stall margin,

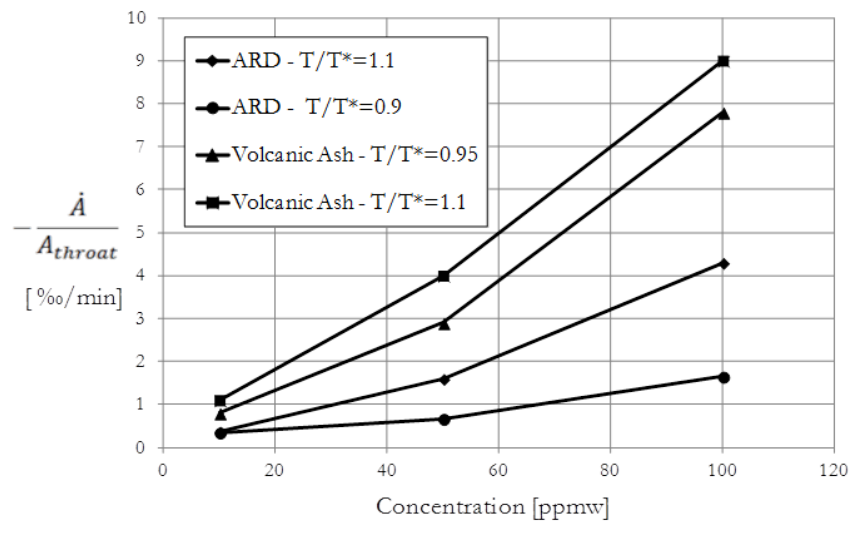

FIGURE 8. Area variation as function of the concentration and reduced temperature

the control system will reduce the load applied to the engine and the fuel flow to the engine. In the worst case scenario the engine will be switched off. This happens if the pressure ratio increases or the non-dimensional flow is reduced. Accordingly to its definition, this last quantity decreases only if $\dot{m}$ or $T_{0}$ decrease, or if $p_{0}$ increases.

The deposition of particles on the first stage HP nozzle can reduce the passage area of the vane. As a consequence, the capacity of the turbine will be reduced moving the compressor operating point towards the instability region (on the left of the surge line). If the reduction in the throat area increases, the compressor working point can cross the surge margin line. To avoid this risk, as suggested by [5], the control system should reduce the TET to a value where the calcium impurity does not melt. The easiest way to do this is to retard the throttle to idle.

The amount of reduction in the passage area which causes the occurrence of such event depends on the specific engine, on the control system and on working parameters. Figure 8 shows the area variation in time as function of the concentration and the relative temperature. The area is reduced increasing the particles in the stream. Graphs obtained with the proposed model can for example be used to estimate how long an engine can flight in a volcanic cloud. Using the concentration of the cloud is possible to estimate the area change per unit of time. This reduces the mass flow of the engine by the same amount because the nozzle throat drives the engine mass flow. With this value is possible to estimate how many seconds/minutes the engine can flight before the compressor is too close to the stall region, using the compressor maps.

\section{Conclusions}

The present work shows an innovative fouling model that uses only the energy content of the particles, based on temperature and kinetic energy, to estimate the sticking probability. It 
is shown that the available data in the open literature verify the proposed law for the variation of the activation energy with the temperature. Though, whatever is the nature of fouling agent (volcanic ashes, sand etc), the sticking phenomenon is only connected to the energy content based on kinetic energy and thermal content, showing for the first time that a single model can characterize any common deposition phenomena in gas turbines, both in the compressor and in the turbine.

The model is implemented into a CFD solver and the well known LS89 test case is modelled. A Lagrangian particle tracking is used and both the CFD solver and the tracking have a parallel implementation. In order to estimate the impact of flow field on particles and the influence of particles on flow structures, both a one way and a two way interaction model between the flow and the particles have been analysed and tested. For the final results a two way implementation is used.

For the LS89 simulation, an iterative approach is used to modify the geometry following the particle deposition. The deposition rate is used to evaluate the airfoil displacement and the geometry is modified accordingly. The mesh is altered and iteratively the flow field is updated by the CFD solver. The formulation is three dimensional and can be implemented in any CFD solver.

The impact of throat area reduction due to fouling it is also estimated. This is a crucial parameter in aircraft engines because it sets the mass flow of the engine and can push the axial compressor towards an unstable regime.

\section{REFERENCES}

[1] Tabakoff, W., 1987. "Compressor erosion and performance deterioration”. Journal of fluids engineering, 109(3), pp. 297-306.

[2] Johnson, R. W., and Casadevall, T. J., 1994. "Aviation safety and volcanic ash clouds in the indonesia-australia region”. In Volcanic ash and aviation safety: Proceedings of the First International Symposium on volcanic ash and aviation safety, Seattle, WA. US Geological Survey Bulletin, Vol. 2047, pp. 191-197.

[3] Smialek, J. L., Archer, F. A., and Garlick, R. G., 1991. The Chemistry of Saudi Arabian Sand: A Deposition Problem on Helicopter Turbine Airfoils. National Aeronautics and Space Administration.

[4] Wenglarz, R., and Fox Jr, R., 1990. "Physical aspects of deposition from coal-water fuels under gas turbine conditions". ASME J. Eng. Gas Turbines Power, 112(1), pp. 914.

[5] Dunn, M. G., 2012. "Operation of gas turbine engines in an environment contaminated with volcanic ash". Journal of Turbomachinery, 134(5), p. 051001.

[6] Ogiriki, E. A., Li, Y. G., Nikolaidis, T., Isaiah, T. E., and Sule, G., 2015. "Effect of fouling, thermal barrier coating degradation and film cooling holes blockage on gas turbine engine creep life". Procedia CIRP, 38, pp. 228-233.

[7] Kurz, R., and Brun, K., 2001. "Degradation in gas turbine systems". Journal of Engineering for Gas Turbines and Power, 123(1), pp. 70-77.

[8] Barker, B., Casaday, B., Shankara, P., Ameri, A., and Bons, J., 2013. "Coal ash deposition on nozzle guide vanespart ii: Computational modeling”. Journal of Turbomachinery, 135(1), p. 11015.

[9] Sreedharan, S. S., and Tafti, D. K., 2010. "Composition dependent model for the prediction of syngas ash deposition with application to a leading edge turbine vane". Proceedings of ASME Turbo Expo 2010: Power for Land, Sea, and Air, pp. 615-626.

[10] Singh, S., and Tafti, D., 2015. "Particle deposition model for particulate flows at high temperatures in gas turbine components". International Journal of Heat and Fluid Flow, 52, pp. 72-83.

[11] Senior, C., and Srinivasachar, S., 1995. "Viscosity of ash particles in combustion systems for prediction of particle sticking". Energy \& Fuels, 9(2), pp. 277-283.

[12] Brach, R. M., and Dunn, P. F., 1992. "A mathematical model of the impact and adhesion of microsphers". Aerosol Science and Technology, 16(1), pp. 51-64.

[13] Elghobashi, S., 1994. "On predicting particle-laden turbulent flows". Applied Scientific Research, 52(4), pp. 309329.

[14] Parker, G., and Lee, P., 1972. "Studies of the deposition of sub-micron particles on turbine blades". Proceedings of the Institution of Mechanical Engineers, 186(1), pp. 519-526.

[15] Delimont, J., Murdock, M., Ng, W., and Ekkad, S., 2014. "Effect of near melting temperatures on microparticle sand rebound characteristics at constant impact velocity". ASME Turbo Expo 2014: Turbine Technical Conference and Exposition.

[16] Crosby, J. M., Lewis, S., Bons, J. P., Ai, W., and Fletcher, T. H., 2007. "Effects of particle size, gas temperature and metal temperature on high pressure turbine deposition in land based gas turbines from various synfuels". Proceedings of ASME Turbo Expo 2007: Power for Land, Sea, and Air, pp. 1365-1376.

[17] Dykhuizen, R., and Smith, M., 1998. "Gas dynamic principles of cold spray". Journal of Thermal Spray Technology, 7(2), pp. 205-212.

[18] Legoux, J.-G., Irissou, E., and Moreau, C., 2007. "Effect of substrate temperature on the formation mechanism of coldsprayed aluminum, zinc and tin coatings". Journal of Thermal Spray Technology, 16(5-6), pp. 619-626.

[19] Morsi, S., and Alexander, A., 1972. "An investigation of particle trajectories in two-phase flow systems". Journal of Fluid Mechanics, 55(02), pp. 193-208.

[20] Mücke, E. P., Saias, I., and Zhu, B., 1996. "Fast random- 
ized point location without preprocessing in two- and threedimensional delaunay triangulations". Proceedings of the Twelfth Annual Symposium on Computational Geometry, pp. 274-283.

[21] M. Carnevale, F. Wang, J. G., and di Mare, L. "Lip stall suppression in powered intakes".

[22] Arts, T., Lambertderouvroit, M., and Rutherford, A., 1990. "Aero-thermal investigation of a highly loaded transonic linear turbine guide vane cascade: a test case for inviscid and viscous flow computations". NASA STI/Recon Technical Report N, 91.

[23] Thompson, J., 1997. "Introduction to "numerical solution of the quasilinear poisson equation in a nonuniform triangle mesh"”. J. Comput. Phys., 135(2), Aug., pp. 126-127.

[24] Taltavull, C., Dean, J., and Clyne, T. W., 2015. "Adhesion of volcanic ash particles under controlled conditions and implications for their deposition in gas turbines". Advanced Engineering Materials.

[25] Gislason, S. R., Hassenkam, T., Nedel, S., Bovet, N., Eiriksdottir, E. S., Alfredsson, H. A., Hem, C. P., Balogh, Z. I., Dideriksen, K., Oskarsson, N., Sigfusson, B., Larsen,
G., and Stipp, S. L. S., 2011. "Characterization of eyjafjallajkull volcanic ash particles and a protocol for rapid risk assessment". Proceedings of the National Academy of Sciences, 108(18), pp. 7307-7312.

[26] Suman, A., Morini, M., Kurz, R., Aldi, N., Brun, K., Pinelli, M., and Spina, P. R., 2014. "Quantitative cfd analyses of particle deposition on a transonic axial compressor blade: Part ii - impact kinematics and particle sticking analysis". American Society of Mechanical Engineers.

[27] Guha, A., 2008. "Transport and deposition of particles in turbulent and laminar flow". Annu. Rev. Fluid Mech, 40, pp. 311-341.

[28] Reitzer, B., 1964. "Rate of scale formation in tubular heat exchangers. mathematical analysis of factors influencing rate of decline of over-all heat transfer coefficients". Industrial \& Engineering Chemistry Process Design and Development, 3(4), pp. 345-348.

[29] Johnson, K., Kendall, K., and Roberts, A., 1971. "Surface energy and the contact of elastic solids". Proceedings of the Royal Society of London A: Mathematical, Physical and Engineering Sciences, 324(1558), pp. 301-313. 